\title{
Aortic Root Enlargement in Patients With Small Aortic Annulus Undergoing Double Valve Replacement: A Retrospective Comparative Cohort Study
}

\author{
Yasser Shaban Mubarak, MD, ${ }^{1,2}$ Ahmed Abdel Rahman Abdel Jawad, MD ${ }^{3,4}$ \\ ${ }^{1}$ Department of Cardiothoracic Surgery, Minia University, Minia, Egypt; ${ }^{2}$ Madinah Cardiac Center, King Fahd Hospital, KSA, Saudi \\ Arabia; ${ }^{3}$ Department of Cardiothoracic Surgery, Cairo University, Cairo, Egypt; ${ }^{4}$ Clinical of Cardiothoracic \& Vascular Surgery, \\ Städtisches Klinikum Braunschweig, Germany
}

\section{ABSTRACT}

Background: Small aortic annulus (AA) is a big issue during aortic valve replacement (AVR), necessitating replacement of an undersized prosthetic valve especially with double valve replacement (DVR). Despite the fact that small aortic valve prostheses can lead to prosthesis-patient mismatch (PPM), there remains reluctance to perform aortic root enlargement (ARE) procedures, fearing morbidity and mortality.

Objective: To evaluate clinical and echocardiographic outcomes in patients with small aortic annulus $(<18 \mathrm{~mm})$ undergoing double valve replacement.

Methods: The study included 100 consecutive patients who underwent DVR for combined rheumatic aortic and mitral valve diseases, between January 2016 and September 2020. Only 50 patients had ARE with DVR. ARE was performed using an autologous or bovine pericardium or Dacron patch by Nick's or Manouguian procedures. The estimated postoperative endpoints were mortality, effective orifice areas (EOA), mean aortic pressure gradient (PG), and valve-related complications. The shortest postoperative follow-up period was 6 months.

Results: The study included 30 male and 70 female patients with mean age of $35 \pm 20$ years, body surface area (BSA) of $1.7 \pm 0.3 \mathrm{~m}^{2}$, aortic annulus diameter was $1.4 \pm 0.4$ $\mathrm{mm}$, aortic orifice area was $0.8 \pm 0.1 \mathrm{~cm}^{2}$, and mean pressure gradient $85 \pm 2.5 \mathrm{mmHg}$. During the follow-up period, there was a mild to moderate paravalvular leak $(1 \%)$ with $1 \%$ heart block and residual gradient on prosthetic aortic valve; this was all in DVR alone.

Conclusion: Enlargement of the aortic root by Nick's or Manouguian technique is safe and effective in patients with small aortic annulus undergoing double valve replacements.

Keywords: Aortic Root Enlargement, Small Aortic Annulus, Double Valve Replacement, Patient-Prosthesis Mismatch, Nick`s / Manouguian procedure.

Received October 19, 2020; received in revised form November 8, 2020; accepted November 9, 2020.

Correspondence: Yasser Shaban Mubarak; +966560708223; +201002554078 (e-mail: yassermubarak73@gmail.com).

\section{INTRODUCTION}

Rheumatic heart disease (RHD) is very common in developing countries. It affects mainly the mitral and aortic valves. It causes annular fibrosis and may lead to smaller AA. So, if a smaller prosthesis is implanted, there may be PPM, poor left ventricle (LV) mass regression, increased afterload, and low survival rate. With an undersized prosthetic valve, a patient cannot maintain normal activity and may produce symptoms of aortic stenosis (AS) and PPM. Rahimtola first described the issue of PPM, if the EOA of prosthetic valve is very small, in relation to a patient's BSA [Ahsan 2020; Ankit 2020]. Nick and colleagues first proposed posterior root enlargement in 1970. Nick's technique facilitated placement of a larger sized aortic valve prosthesis by extending the aortotomy posteriorly through aortic sinus across to aortic ring and inserting a patch to augment the annulus [Grubb 2016].

Severe PPM, according to the valve's EOA indexed (EOAi) [severe PPM when an EOAi $<0.65 \mathrm{~cm}^{2} / \mathrm{m}^{2}$ ], is associated with worse hemodynamic and clinical outcome [Rocha 2018]. PPM is a predictor of mortality and it can be responsible for postoperative high transvalvular PG. ARE allows for larger prosthesis implantation, consequently avoiding PPM. Despite these potential benefits of ARE, it has not been widely performed by cardiac surgeons, fearing increased risk of mortality and morbidity [Sa 2019; Timala 2010; Fuster 2005].

Cardiac surgeons performing AVR should be familiar with the techniques of ARE to allow insertion of appropriate-sized prostheses in case of small AA and to avoid PPM. When ARE was performed properly by an experienced cardiac surgeon, the technique is safe and reproducible [Feindel 2006]. So, nowadays surgeons prefer to do ARE to get rid of PPM and to obtain optimum hemodynamics [Ahsan 2020; Ankit 2020].

\section{METHODS}

Demographic, intraoperative, and outcome data retrospectively were collected for a cohort comparative study on 100 patients undergoing DVR with or without ARE at multicenter institutions between January 2016 through September 2020. Those patients with pure or predominant aortic valve stenosis were included in the study. One-hundred patients with small AA had DVR performed. Fifty of these patients underwent ARE with DVR, and the other 50 did not undergo 


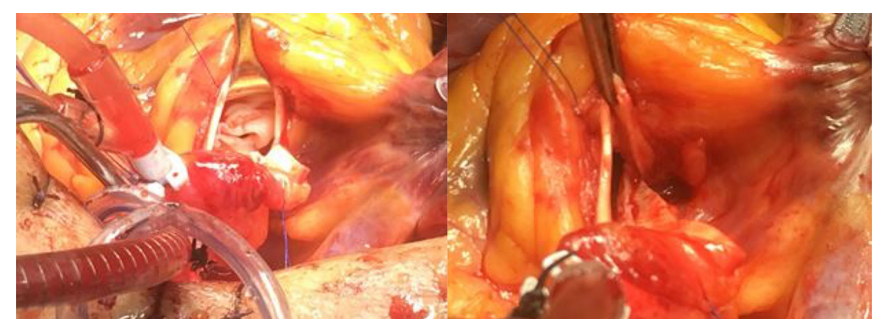

Figure 1. Nick's procedure by incision of non-coronary cusp.

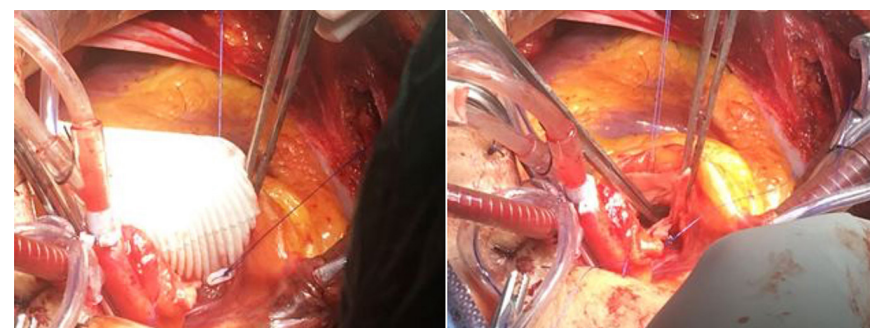

Figure 2. Patch suture started at angle of incision.

ARE, due to lack of surgeons experience. Mean age was $35 \pm$ 20 years. Operative death and residual gradient on prosthetic aortic valve $(A V)$ were evaluated. Inclusion criteria included RHD adult patients who underwent elective DVR and ARE, due to small aortic annulus. Exclusion criteria: Patients who underwent only AVR or other than RHD. Patients excluded from study included cases of emergency, redo, renal failure, children, elder or procedures other than DVR.

A median sternotomy was performed in all patients. Cardiopulmonary bypass (CPB) with systemic cooling to $32^{\circ} \mathrm{C}$ was routinely used. After the aorta was cross-clamped (Ao.CC) and the heart is arrested by means of intermittent, antegrade cold blood cardioplegia directly was delivered into the coronary ostia. The decision to maximize EOA was made after intraoperative assessment of AA. After debridement of the aorta annulus, its size not suitable to BSA, aortotomy incision was extended into the fibrous trigone between NCC and LCC [Manouguian] or extended into NCC [Nick's] to enlarge AA. This incision was reconstructed using a tear-drop-shaped patch of autologous pericardium [pericardial patch was harvested and fixed with glutaraldehyde] or bovine pericardium or Dacron patch. The patch was sutured with $4 / 0$ polypropylene, starting at the nadir of annular enlargement incision and extending up to $2-3 \mathrm{~cm}$ above the plane of annulus. After replacing the mitral valve, AA was resized and the appropriate valve was chosen. We use a non-everting, horizontal mattress technique of $2 / 0$ polyester placed on annulus. Pledgeted sutures were placed in the plane of annulus, where patch enlargement was performed with pledgets resting on the outside of the patch. After replacing the valve patch, it was sutured to aortotomy margins using 4/0 polypropylene.

Simplified Manouguian is allowed with ARE without opening LA, without distorting the mitral annulus, and decreased incidence of potential bleeding. Also, modified Nick's procedure is allowed with ARE without extended to mitral annulus and can be performed in DVR.

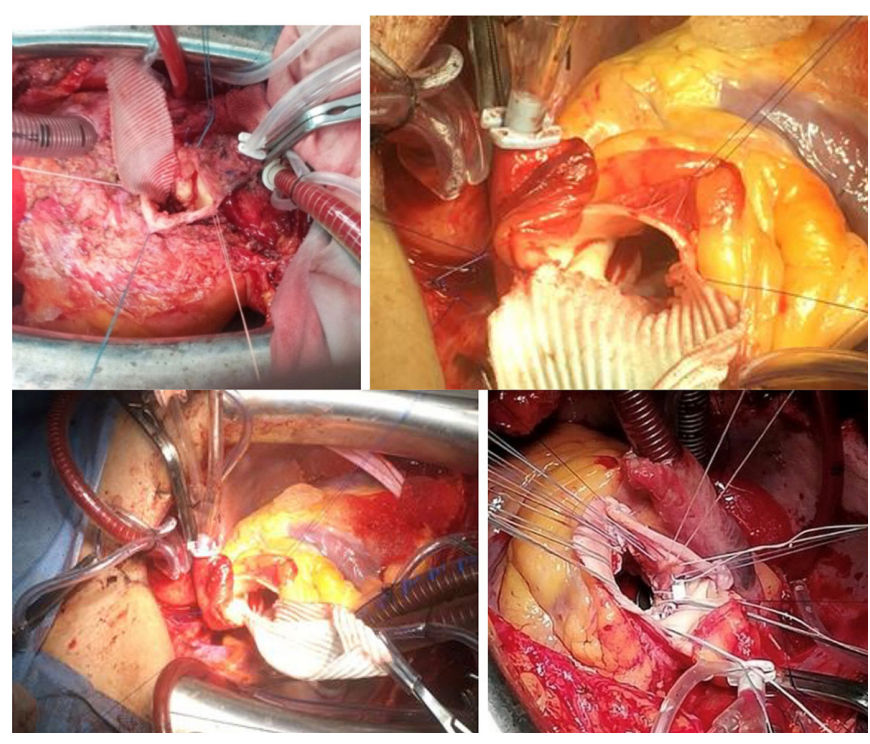

Figure 3. Complete patch suture and suture for valve taken.

In cases of small $\mathrm{AA}$ accepted small prosthetic $\mathrm{AV}$ without ARE, we have started to implant AV after putting pledgets along the mitral annulus before im $\neg$ planting AV. So, AA gets the size of the valve that it deserves. Then, one easily can implant the mitral valve. For better hemodynamic effect, we prefer to implant the prosthetic $\mathrm{AV}$ in the anteroposterior direction. We prefer to implant prosthetic mitral valve in anatomical position, so that struts of mitral valve do not impinge upon AV, which is already in position (Figures 1, 2, 3).

\section{RESULTS}

Demographic data shows that commonalities between the two groups include young adult age and the incidence of obese females. RHD is more common in young females. There is no significant difference in preoperative risk factors in both groups. Aortic stenosis is a predominate lesion in the ARE group (Table 1).

Postoperative echocardiogram (Echo) before discharge showed acceptable gradients across the aortic valve in all cases with ARE, however there are PMM with cases of DVR only. We encourage them to decrease body weight and do a followup Echo (Table 2).

The duration of aortic cross-clamp is slightly longer by approximately 10 minutes compared with DVR alone; it didn't make a significant difference in overall management of the patient. There is no incidence of intra-operative or postoperative bleeding, no excessive requirement of blood products. There was mild to moderate paravalvular leak in $1 \%$ and heart block in $1 \%$ in DVR without ARE. Also, there is no significant difference in infection, ICU stay, and total hospital stay (Table 3).

We used St. Jude Medical Standard (mechanical valves) implanted intra-annular in all cases of study. With root enlargement one-to-two sizes bigger were replaced in 50 patients, and PPM was eliminated in all patients. Nick's was 
Table 1. Demographic data, pre-operative risk factors

\begin{tabular}{|c|c|c|c|}
\hline Variable & $\begin{array}{l}\text { DVR alone } \\
(N=50)\end{array}$ & $\begin{array}{l}\text { DVR with ARE } \\
\qquad(N=50)\end{array}$ & \\
\hline Age & Mean $32 \pm 24$ & Mean $35 \pm 20$ & \\
\hline Gender & $65 \%$ female & $70 \%$ female & \\
\hline BSA & $1.7 \pm 0.3 \mathrm{~m}^{2}$ & $1.6 \pm 0.4 \mathrm{~m}^{2}$ & \\
\hline \multicolumn{4}{|l|}{ NYHA class } \\
\hline 1 & $5.9 \%$ & $4.1 \%$ & \\
\hline 2 & $19.8 \%$ & $15.9 \%$ & \\
\hline 3 & $60.4 \%$ & $68.5 \%$ & \\
\hline 4 & $13.9 \%$ & $11.5 \%$ & \\
\hline $\mathrm{AF}$ & $20 \%$ & $30 \%$ & \\
\hline PVD & No & No & \\
\hline COPD & No & No & \\
\hline LCO & No & No & \\
\hline HTN & No & $0.5 \%$ & \\
\hline DM & No & $2.5 \%$ & \\
\hline $\mathrm{s} / \mathrm{p} \mathrm{MI}$ or $\mathrm{CAD}$ & No & No & \\
\hline Smoking & $5 \%$ & $10 \%$ & \\
\hline Stroke and TIAs & No & No & \\
\hline \multicolumn{4}{|l|}{ Aortic lesion } \\
\hline Stenosis & $18.4 \%$ & $34 \%$ & $P \leq .0001$ \\
\hline Insufficiency & $10.8 \%$ & $3.7 \%$ & .0002 \\
\hline Mixed & $70 \%$ & $61.6 \%$ & .0069 \\
\hline
\end{tabular}

The statistical analysis was performed using the SPSS software package (version 20.0; SPSS Inc., Chicago, IL, USA). The analyzed data were expressed as number $(\mathrm{N})$, percentage (\%), mean (M) and standard deviation (SD) or as proportions. $P$-value $<.05$ was considered statistically significant.

Table 2. Pressure gradient and EF

\begin{tabular}{lcc}
\hline Variable & DVR alone & DVR with ARE \\
\hline Preop. mean PG (mmHg) & $75 \pm 3.5$ & $85 \pm 2.5$ \\
Postop. mean PG (mmHg) & $28.9 \pm 3.8$ & $15 \pm 2.3$ \\
EF (\%) Postop. & $45 \pm 4.7$ & $55 \pm 5.9$
\end{tabular}

performed in 45 cases and Manouguian in 5 cases. Type of patches used include autologous pericardium in 35 cases, Dacron in 12 cases, and Bovine in 3 cases. There was no operative or in-hospital mortality (Table 4).

\section{DISCUSSION}

Patient age and activity can be considered for calculating cardiac output demand; young people with an active
Table 3. Postoperative data

\begin{tabular}{lcc}
\hline Variable & DVR alone & DVR with ARE \\
\hline CPB time (minutes) & $117 \pm 43$ & $133 \pm 49$ \\
CC time (minutes) & $92 \pm 35$ & $105 \pm 39$ \\
Postop. LCO & No & $4 \%$ \\
Bleeding and re-exploration & No & No \\
Blood product requirement & $2 \pm 1.5$ & $3 \pm 2.3$ \\
Duration of MV (hr.) & $12 \pm 6$ & $18 \pm 6.4$ \\
Infection & & \\
1 - SSWI & $1.5 \%$ & $3 \%$ \\
2 - Pneumonia & No & $0.5 \%$ \\
Heart block (HB) & $1 \%$ & No \\
Paravalvular Leak (PVL) & $1 \%$ & No \\
ICU stay (days) & $3 \pm 1.8$ & $4 \pm 2.3$ \\
Total hospital stay (days) & $10 \pm 5.1$ & $12 \pm 7.2$
\end{tabular}

Table 4. Size of prosthetic valves

\begin{tabular}{lcc} 
& AVR alone & AVR with ARE \\
\hline Prosthetic aortic valve & 19 & $-23-25$ \\
Prosthetic mitral valve & $25-27$ & $27-29$
\end{tabular}

lifestyle require larger prosthesis for a higher cardiac demand. Implanting a small-sized valve can worsen outcome, due to increased preload [Ankit 2020]. This study was undertaken to review our strategy and feasibility of ARE in patients undergoing DVR to avoid PPM without increase in morbidity or mortality especially in young patients (age $=35 \pm 20$ ).

In patients with a small AA, it is difficult to implant large valve prostheses. PPM is the immediate consequence of this situation [Timala 2010]. We observed high variable pressure gradient across aortic prosthesis with DVR alone (postoperative mean PG $28.9 \pm 3.8$ ).

Rheumatic heart diseases usually affect left heart valves requirement DVR. Small aortic annulus is a big problem facing cardiac surgeons in AVR and more with DVR. Most of the patients also have tricuspid valve disease, atrial fibrillation, and severe LV dysfunction that add risk factors on hemodynamics if ARE is not performed. So, there still is debate as to whether small prosthesis implants or ARE avoid increasing morbidity or mortality [Muppiri 2011]. In our study, preoperative risk factors were not obstacles to perform ARE, however, these factors may be worse with PPM.

ARE techniques can be performed simply and modified without complexity to gain benefits and avoid complications. So, that is an alternative to implantation of small prosthesis, ARE may actually reduce mortality [Sundt 2006]. Our study used to perform modified Nick's procedure to implant larger prosthesis without increasing risk of technique even with 
junior surgeons.

There have been few studies on DVR with ARE. Some are with only a small number of patients of non-Rheumatic etiology; most are case reports. ARE in DVR is enlarging AA without increase in operative mortality but at the expense of prolonged CPB time [Sundt 2006]. That encourages us to collect data for comparison between the two groups of DVR with/out ARE, and motivate cardiac surgeons to ARE, if needed to avoid PPM. ARE itself does not increase operative risk. Surgeons should not be reluctant to enlarge the aortic root to permit implantation of an adequately-sized valve prostheses.

ARE requires some technical skills and should not increase operative risk. So, it is possible to implant a valve two sizes larger than the native annulus [Rocha 2018]. We observed in the study no incremental risk in mortality or adverse events after surgical ARE compared with AVR alone.

Most surgeons prefer to use a small aortic prosthesis instead of expanding the annulus. Yet the use of a small aortic prosthesis may be associated with obstruction of left ventricular output, resulting in a higher PG and PPM. Studies have demonstrated that mortality was higher in patients receiving a small aortic prosthesis [Wang 2013]. So, ARE is a safe procedure in the hands of an expert surgeon and should be considered at the time of AVR even with DVR to avoid PPM.

Surgical ARE has not widely been performed by cardiac surgeons because of concerns, regarding the possible increased risk of early mortality and morbidity [Rocha 2018]. In our study, ARE was safe and did not increase morbidity and mortality.

Any degree of PPM significantly decreases long-term survival and increases readmission rates. ARE and new generation valve designs (supra-annular position, regent valve) all emphasize increasing EOA and reducing PPM. Although the rates of moderate PPM also have decreased, this change has not happened to the same extent as in severe [Fallon 2018]. In our study, we need long-term follow up to evaluate survival.

\section{CONCLUSION}

Aortic root enlargement can be safely done in patients undergoing double valve replacement with the benefit of a bigger size prosthesis without additional mortality and morbidity.

\section{Abbreviations}

ARE: aortic root enlargement, DVR: double valve replacement, AV: aortic valve, AVR: aortic valve replacement, AA: Aortic Annulus, NCC: Non Coronary Cusp, LCC: Left Coronary Cusp, LV: Left Ventricle, RHD: rheumatic heart disease, PPM: Prosthesis Patient Mismatch, EOA/i: Effective
Orifice Area/ indexed, RHD: Rheumatic Heart Disease, BSA: Body Surface Area, PG: Pressure Gradient, EF: Ejection Fraction, CPB: Cardio Pulmonary Bypass, Ao. /CC time: Aortic Cross Clamp/ time, MV: Mechanical Ventilation, HB: Heart Block, LCO: Low Cardiac Output, COPD: Chronic Obstructive Pulmonary Disease, CAD: Coronary Artery Disease, DM: Diabetes Mellitus, HTN: Hypertension, AF: Atrial Fibrillation, AS: Aortic Stenosis.

\section{REFERENCES}

Ahsan M, Rahman L, Shariful A, Arifur M. 2020. Case Report: Aortic Root Enlargement In Case of Double Valve Replacement. KYAMC Journal. 11(2): 108-10.

Ankit M, Ravi G, Debmalya S, et al. 2020. Konno Procedure for Managing Small Aortic Root during Aortic Valve Replacement Surgery: An Experience of 12 Cases. WJ Cardiovasc Surg. 10: 24-31.

Fallon JM, DeSimone JP, Brennan JM, O’Brien S, Thibault DP, DiScipio $\mathrm{AW}$, et al. 2018. The incidence and consequence of prosthesis patient mismatch after surgical aortic valve replacement. Ann Thorac Surg. 106:14-23.

Feindel CM. 2006. Aortic Root Enlargement in the Adult. 03.002. doi:10.1053/j.optechstcvs.2006.03.002

Fuster RG, Argudo JA, Albarova OG, et al. 2005. Patient-prosthesis mismatch in aortic valve replacement: really tolerable? Euro J Cardiothorac Surg. 27: 441-49.

Grubb KJ. 2016. Aortic Root Enlargement during Aortic Valve Replacement: Nicks and Manouguian Techniques. Operative Techniques in Thorac Cardiovasc Surg. 20: 206-18.

Muppiri VK, Ali SN, Venkat KR, Rama KL. 2011. Aortic root enlargement in patients undergoing double valve replacement for rheumatic etiology-preliminary results. Indian J Thorac Cardiovasc Surg. 27(4):161-64.

Rocha R, Manlhiot C, Feindel C, Yau T, Mueller B, et al. 2018. Surgical Enlargement of the Aortic Root Does Not Increase the Operative Risk of Aortic Valve Replacement. Circulation. 137:1585-94.

Sa ${ }^{\prime}$ MPBO, Carvalho MMB, Sobral Filho DC, Cavalcanti LRP, Diniz RGS, et al. 2019. Impact of surgical aortic root enlargement on the outcomes of aortic valve replacement: a meta-analysis of 13174 patients. Interact CardioVasc Thorac Surg. doi:10.1093/icvts/ivy364.

Sundt TM. 2006. Patch Enlargement of the Aortic Annulus using the Manouguian Technique. 03.003.

Timala RB. 2010. How I do it" Aortic Valve Replacement in case of Double Valve Replacement. NHJ. 7(1):50-53.

Wang B, Yang H, Wu S, Cao G, Yang H. 2013. Obesity and the risk of late mortality after aortic valve replacement with small prosthesis. J Cardiothorac Surg. 8: 174 - 75. 